\title{
THE SURGERY OF PORTAL HYPERTENSION
}

\author{
By R. Milnes Walker, M.S.Lond. \\ Professor of Surgery, University of Bristol
}

The renewed interest which has taken place during the past half decade in the relief of portal hypertension by surgical means has called for precise methods of diagnosis, both of the cause and of the degree of portal hypertension. Knowledge of the normal variations in the portal blood pressure in man is still inadequate, and it is probable that an elevation considerably above the normal can occur without any deleterious effects, for in all patients who show clinical evidences of the condition, the figure, when measured, is found to be well above that commonly found in patients on whom an abdominal operation is being performed for some other condition.

In practice it is rare to find a patient in whom the portal hypertension does not affect the whole of the portal system and the surgical treatment discussed here refers only to such cases. The obstruction is either in the liver itself, the result of cirrhosis of the liver, or of thrombosis of the portal vein. The effects are identical, but in the case of cirrhosis not only are the effects of the portal hypertension evident, but also the effects of the disturbed liver function.

The cases in which there is thrombosis of the portal vein are mainly of congenital origin, and haematemesis is the only symptom; the first attack occurs during the early years of life and, if untreated, this bleeding is eventually fatal. The liver in such cases is normal both on gross examination and histologically, but it is these patients who have the highest portal pressure, usually over $400 \mathrm{~mm}$. of water and, as this bleeding from oesophageal varices is the only source of danger, all possible steps to prevent it should be considered.

The normal portal pressure is about $100 \mathrm{~mm}$. of water, and readings above $150 \mathrm{~mm}$. must be considered excessive. In the case of cirrhosis of the liver we have found readings up to $360 \mathrm{~mm}$. and, in congenital obstruction to the portal vein, up to $490^{\circ} \mathrm{mm}$., while other observers have recorded even higher readings. It is important to emphasize that the degree of ascites bears no relation to the portal venous pressure and, if the liver is normal histologically, ascites is never present.

\section{Diagnosis}

- The surgeon may be called upon to assist in the diagnosis in one of four ways:

\section{(I) Oesophagoscopy}

The presence of varicose veins in the lower part of the oesophagus is a definite sign of portal hypertension. Though such varíces can usually be demonstrated by an X-ray during a barium swallow, they may be missed, and in suspicious cases an oesophagoscopy should be performed; in the milder cases they show as rounded bluish protuberances of the mucosa of the oesophagus, while in the more severe examples they closely resemble internal haemorrhoids as seen through the proctoscope.

\section{(2) Peritoneoscopy}

In occasional cases, particularly those with ascites, this investigation may be of value. The appearance of the surface of the liver can be studied, and enlarged veins in the falciform ligament and on the surrounding peritoneal surface of the abdominal wall may be evident long before they appear in the subcutaneous tissue around the umbilicus.

\section{(3) Liver Biopsy}

Though liver puncture may give the information required about the histology of this organ, if a laparotomy has to be done, the removal of an adequate specimen for biopsy has much to commend it. The degree of inflammatory change then demonstrated may be quite considerable, even in a liver which looks and feels almost normal.

\section{(4) Portal Pressure Readings}

An accurate determination of the portal blood pressure can only be made by direct measurement with a needle, canula or polythene catheter inserted into one of the veins of the portal system. Though the portal vein itself or the splenic vein may be used for this purpose, these deeply situated veins are apt to give inconstant readings, owing to pressure on them during the necessary manipulations. It is therefore better to use one of the veins of the mesentery of the jejunum for this purpose. 


\section{Indications for Surgery}

Probably half the sufferers from portal hypertension die as a result of bleeding from the varicose veins in the lower part of the oesophagus, and the aim of surgery is to avert this danger. The collateral circulation which develops at the other end of the alimentary tract as internal haemorrhoids is rarely a cause for anxiety. It is very unusual for patients to succumb to their first haemorrhage from the oesophagus, but one severe bleeding is a red flag of danger, and is a strong indication for surgical intervention. After such a haemorrhage, operation should be advised in all patients except the elderly, those with severe deficiency of liver function or those with some other disease which renders operation particularly hazardous.

It is doubtful whether ascites is a direct result of portal hypertension, and if this rather than haematemesis is the presenting symptom in a patient who has portal hypertension, there is some degree of hepatic deficiency and surgical treatment should only be embarked on with caution. Ascites is, moreover, a symptom which shows great spontaneous variation, and the influence of operative measures on the amount of ascitic fluid can only be assessed after long experience and a comparison with control cases.

The suggestion has been made that the relief of portal hypertension by means of a venous shunt will have a beneficial effect on the pathological condition in the liver when the hypertension is associated with chronic hepatitis. There is no conclusive evidence for this, and further speculation must await a more intimate knowledge of the hepatic circulation. There is, however, some evidence that the pressure is transmitted not only from the gastric and mesenteric arteries through their corresponding capillary bed, but also from the hepatic artery through its connections with the portal vein in the liver.

\section{Surgical Procedures}

The surgical methods adopted in portal hypertension may be classified as follows:

(I) Methods which aim at reducing the portal pressure.

(a) By reducing the blood entering the portal system:-

(i) Splenectomy.

(ii) Splenic artery ligation.

(b) By promoting collateral circulation.

(i) By promoting vascular adhesions.

(ii) By a direct venous anastomosis.

(2) Methods which divert the blood from the oesophageal varices.

(a) Direct attack on the varices.

(i) Injection and thrombosis of the varices. (ii) Excision of the varices.

(iii) Multiple ligation of the varices. (b) Dividing vessels leading to the varices.

These methods will be considered in turn.

(i) Reducing the Portal Pressure

(a) By Reducing the Blood Entering the Portal System

(i) Splenectomy. This operation has been practised frequently in cases of portal hypertension without gross fibrosis in the liver. Howell (1938) in a study of 94 patients, amongst which 57 splenectomies were carried out, came to the conclusion that there was no significant difference in the survival rates when those who had a splenectomy were compared with those treated without operation. In a case of our own, in which splenectomy was carried out on account of haemorrhage, slight further bleeding occurred after a symptom-free interval of ten years, and a more severe haematemesis three years later. In many cases, however, the symptom recurs only too soon after this operation. Splenectomy may possibly have an adverse effect, for many of these patients have developed a considerable collateral circulation between the splenic vessels and those of the posterior $a b_{-}$ dominal wall, particularly in the lieno-renal ligament, and the operation necessarily entails the re moval of this circulation which it is most im portant to preserve.

(ii) Splenic Artery Ligation. This operation has been advocated in poor risk patients. It has the advantages of simplicity and that it does not remove any of the collateral circulation, but its effectiveness has been doubted. Blair and Blair (1950) have recorded a patient who survived the operation for 34 years, but unfortunately do not give details of the pathology. Watson (1935) seems to have been the first to undertake it deliberately for portal hypertension as a result of a suggestion by McNee (193I), and his patient was much improved when reported three years later.

Eight of our patients have had this operation performed more than six months ago, but in two there has been recurrence of bleeding.

\section{(b) By Promoting Colbateral Circulation}

(i) By promoting vascular adhesions. The TalmaMorison operation in one form or another is the method by which this is done, promoting adhesions between the liver and spleen and the parietal peritoneum and implanting the great omentum into the anterior abdominal wall. Though possibly of value in cases of severe chronic hepatitis, its use is limited and it should only be employed when all other methods are impracticable.

(ii) By a direct venous anatomosis. This type of operation has been brought to the fore as a result 
of the work of Whipple (1945) and of Blakemore and Lord (1945). Whipple has summarized the literature of the earlier attempts at direct venous anastomosis, while the other authors described a technique using a vitallium tube, though they themselves have now adopted an intima-tointima suture technique which is now generally employed for vascular anastomoses.

The site of the anastomosis depends on the findings at operation, and which vessels are suitable. Most commonly employed is an end-to-side union between the splenic vein and the left renal vein after removal of the spleen. The advantages are that often the splenic vein is enlarged and so a wide opening can be effected, and that these veins naturally lie close to each other. The tail of the pancreas may be in the way and will then require free mobilization. The disadvantages are that the preliminary splenectomy may be difficult on account of vascular adhesions, and that the renal vein is deeply situated, so that the suturing has to be done in a deep wound, and adequate access may be impossible in a stout patient.

In order to preserve the collateral circulation already existing round the spleen, we have, in these cases, left the spleen untouched, but divided the splenic artery and made a side-to-side anastomosis between the splenic and left renal veins. It is, however, not always possible to approximate these two veins while in continuity.

Another site for anastomosis is a direct union between the portal vein and the inferior vena cava, either side-to-side or end-to-side after division of the portal vein. Various special instruments have been devised for this operation so that the suturing can be performed without occluding the whole lumen of the inferior vena cava. Its application is limited, for the portal vein may be obliterated by thrombus or replaced by numerous tortuous channels. The inferior vena cava is deeply situated, and though a right transthoracic approach may facilitate the operation, again in a stout patient inadequate access may prevent this operation being done.

Occasionally it may be convenient to use other veins for a portal-systemic anastomosis. Thus in one patient who had large veins in his anterior abdominal wall as a result of an old thrombosis of his inferior vena cava, the left gastric vein was joined to one of these venous channels in the abdominal wall.

(2) Diverting the Flow of Blood from the OESOPHAGEAL VARICES

(a) Direct Attack on the Varices

(i) Injection of the veins with a sclerosing solution throught the oesophagoscope. Introduced by Crafoord and Frencker (1939) this method can hardly be expected to prevent further haemorrhage when the extent of the varices in the severe cases is seen. It is rarely practised nowadays.

(ii) Excision of the Varices. Phemister and Humphreys (1947) reported two patients in whom they had resected the varicose vein-bearing area. Both cases had had a previous splenectomy; in one a total gastrectomy and in the other an oesophago-gastrectomy was performed. The first case had had two further haemorrhages when he was reported two years after his operation, and this seems an unnecessarily drastic operation which, at the best, can only be a palliative measure.

(iii) Multiple Ligation and Injection of the Varices. Boerema (1949) records two patients in whom he exposed the oesophagus through a left thoracotomy, opened the oesophagus, and tied multiple under-running sutures round the dilated veins, subsequently injecting a sclerosing solution into the veins between his sutures. Like the previous method, this is a drastic procedure, and again it can only be palliative.

\section{(b) Dividing Vessels Leading to the Varices}

This rational procedure, which aims at diverting the collateral circulation into safer channels, has in its most recent form been suggested by Tanner (I950). The operation consists in division of the left gastric vein and the vasa brevia; the stomach is then transected high up and re-sutured. The left gastric artery is retained in order to preserve the blood supply to the fundus of the stomach. Such an operation, again, can only be palliative, for new venous channels will undoubtedly form, but it has the merit that it can be performed when other methods have failed. We have used it on two occasions, both children with extra-hepatic portal vein obstruction; one had a splenectomy performed five years previously, and the other division of the splenic artery a year before, and in both instances there had been a recurrence of haemorrhage.

With the exception of patients who have severely impaired liver function, we believe that a laparotomy is to be recommended in all patients with portal hypertension who have had one or more severe haemorrhages from oesophageal varices.

The surgeon who undertakes this laparotomy must be prepared to carry out a venous anastomosis if the findings render this possible, as it is likely that the performance of such a by-pass is the surest way of preventing further bleeding. Preparations must therefore be made for a long and tedious operation and adequate blood for infusion must be at hand. After trying many approaches, we find that for the initial exploration an upper midline incision is the most satisfactory. After a preliminary examination of the liver and spleen, the 
region of the portal vein in inspected and palpated, but at this stage no attempt is made to dissect out the vein. After taking a biopsy from the liver, the portal pressure is measured by passing a polythene tube connected to a manometer into a suitable tributary of the portal vein, usually one in the mesentery of the jejunum. The lesser sac is then opened and the splenic vessels examined. A decision must now be reached regarding the procedure to be carried out. At the moment we favour a side-to-side anastomosis between the splenic and renal veins, with division of the splenic artery. This is most conveniently done through the lesser sac after mobilization of the tail of the pancreas. However, if there is a wide splenic vein and the spleen is free from adhesions, a splenectomy and end-to-side anastomosis will serve the purpose, and has the advantage of allowing more mobilization of the splenic vein, so that this may be possible in some cases when a side-toside union cannot be done. If the spleen has been removed at a previous operation, the choice is limited and the portal vein will have to be dissected out, to see whether it is patent and suitable for an anastomosis, if such a procedure is feasible. Access from in front is unsatisfactory, however, and it is wiser to close the wound and, on another occasion, approach the site by means of a right thoractomy with incision of the diaphragm, as recommended by Satinsky (1948).

If no venous anastomosis is practicable, one of the less radical measures should be undertaken. At the moment we are following up a series in which the splenic artery has been divided, either alone or combined with division of the left gastric vein, for in these cases it can be demonstrated that the blood in this vein is flowing in a reversed direction, i.e. away from the portal vein. This manoeuvre will cause a fall in portal pressure of between $5^{\circ}$ and $100 \mathrm{~mm}$. of water, but from experience we know this may not be enough to prevent further bleeding. As an alternative, the operation recommended by Tanner can be combined with splenic artery division.
In our own series, if we exclude patients operated on less than six months ago, 33 laparotomies have been undertaken. In 15 a vascular anastomosis has been performed, with 3 deaths as a result of consequences of the operation. The remaining 12 are all alive for periods up to three years, but some have symptoms as a result of poor liver function; one, a child who had had a previous splenectomy, and for whom a porta-caval anastomosis was performed, has had a further slight bleeding.

Eight patients have had a splenic artery ligation, and two of them have had further haemorrhage, for one of whom the operation recommended by Tanner has since been carried out.

Six patients had splenectomy alone early in the series; two died from complications of the operation, two died as a result of further haemorrhage two and three years respectively after the splenectomy, and the other two have both had further bleeding.

Various other procedures were carried out in the remaining four patients, two of whom had already had their spleens removed and had had a recurrence of haemorrhage.

From this small series, it seems therefore that a venous shunt between the portal and systemic circulations gives the best chance for the patient of avoiding further haemorrhage, but that where such an operation cannot be carried out, there are less radical measures which may postpone a fataF termination.

\section{BIBLIOGRAPHY}

- BLAKEMORE, A. H., and L'ORD, T. W. (1945), Ann. Surg., I22, 476.

- BOEREMA, I. (1949), Arch. Chir. Neerland, r, 253.

CRAFOORD, C., and FRENCKER, P. (1939), Acta Otolaryng., 27, 422 .

HOWELLS, L. (1938), Lancet, I, 1320.

McNEE, T. W. (193I), Lancet, I, I069.

PHEMISTER, D. B., and HUMPHREYS, E. M. (1947), Ann. Surg., 126, 397.

SATINSKY, V. P. (1948), Ann. Surg., 128, 938.

- TANNER, N. C. (1950), Proc. Roy. Soc. Med., 43, 147.

WATSON, R. B. (I735), Brit. Med. F., I, 821 .

- WHIPPLE, A. O. (1945), Ann. Surg., 122, 449. 